Portland State University

PDXScholar

\title{
Complex Analysis of Intracranial Hypertension Using Approximate Entropy
}

\author{
Roberto Hornero \\ University of Valladolid \\ Mateo Aboy \\ Portland State University \\ Daniel Abasolo \\ University of Valladolid \\ James McNames \\ Portland State University \\ Wayne Wakeland \\ Portland State University, wakeland@pdx.edu
}

See next page for additional authors

Follow this and additional works at: https://pdxscholar.library.pdx.edu/sysc_fac

Part of the Medicine and Health Sciences Commons

Let us know how access to this document benefits you.

\section{Citation Details}

Hornero, Roberto; Aboy, Mateo; Abasolo, Daniel; McNames, James; Wakeland, Wayne; and Goldstein, Brahm, "Complex Analysis of Intracranial Hypertension Using Approximate Entropy" (2006). Systems Science Faculty Publications and Presentations. 70.

https://pdxscholar.library.pdx.edu/sysc_fac/70

This Post-Print is brought to you for free and open access. It has been accepted for inclusion in Systems Science Faculty Publications and Presentations by an authorized administrator of PDXScholar. Please contact us if we can make this document more accessible: pdxscholar@pdx.edu. 


\section{Authors}

Roberto Hornero, Mateo Aboy, Daniel Abasolo, James McNames, Wayne Wakeland, and Brahm Goldstein 


\title{
COMPLEX ANALYSIS OF INTRACRANIAL HYPERTENSION USING APPROXIMATE ENTROPY
}

\author{
Roberto Hornero, $\mathrm{PhD}^{1}$ \\ Mateo Aboy, $\mathrm{MS}^{2,3}$ \\ Daniel Abasolo, $\mathrm{MS}^{1}$ \\ James McNames, $\mathrm{PhD}^{2}$ \\ Wayne Wakeland, Ph.D ${ }^{4}$ \\ Brahm Goldstein, MD, FCCM ${ }^{5}$ \\ ${ }^{1}$ ETSI-Telecomunicación de Valladolid, University of Valladolid, Spain, EU \\ ${ }^{2}$ Biomedical Signal Processing Laboratory, Portland State University, USA \\ ${ }^{3}$ ETSI-Telecomunicación de Vigo, University of Vigo, Spain, EU \\ ${ }^{4}$ Systems Science Ph.D. Program, Portland State University, USA \\ ${ }^{5}$ Division of Pediatric Critical Care, Pediatrics, Oregon Health \& Science University, USA
}

\author{
Address for Correspondence: \\ Brahm Goldstein, MD, FCCM \\ Professor of Pediatrics \\ Director, Complex Systems Laboratory \\ Doernbecher Children's Hospital \\ Oregon Health \& Science University \\ 707 SW Gaines St. \\ Mail Code CDRCP \\ Portland, OR 97239 \\ Tel. 503-418-5177 \\ Fax 503-494-6052 \\ Email: goldsteb@ohsu.edu
}




\section{ABSTRACT}

Objective: To determine if decomplexification of intracranial pressure (ICP) dynamics occurs during periods of severe intracranial hypertension (ICP $>25 \mathrm{mmHg}$ for longer than 5 minutes in the absence of external noxious stimuli) in pediatric patients with intracranial hypertension.

Design: Retrospective analysis of clinical case series over a 30-month period from April 2000 through January 2003.

Setting: Multi-disciplinary 16-bed Pediatric Intensive Care Unit.

Subjects: Eleven episodes of intracranial hypertension from 7 subjects requiring ventriculostomy catheter for intracranial pressure monitoring and/or cerebral spinal fluid drainage.

Intervention: None.

Measurements and Main Results: We measured changes in the ICP complexity, estimated by the approximate entropy $(\mathrm{ApEn})$ as patients progressesed from a state of normal ICP $(<25 \mathrm{mmHg})$ to intracracranial hypertension. We found the ApEn population mean to be lower during the intracracranial hypertension period than during the stable and recovering periods in all the 11 episodes $(0.5158 \pm 0.0089$, $0.3887 \pm 0.077$, and $0.5096 \pm 0.0158$, respectively, $\mathrm{p}<0.01$ ). Both the mean reduction in ApEn from the state of normal ICP (stable region) to intracranial hypertension (-0.1271), and the increase in ApEn from the ICH region to the recovering region $(0.1209)$ were determined to be statistically significant $(\mathrm{p}<0.01)$. Conclusions: Our results indicate that decreased complexity of ICP coincides with periods of intracranial hypertension in brain injury. This suggests that the complex regulatory mechanisms that govern ICP may be disrupted during acute periods of intracranial hypertension. This phenomenon of decomplexification of physiologic dynamics many have important clinical implications for ICP management.

KEY WORDS: brain injury, intracranial pressure, intracranial hypertension, approximate entropy, complexity 


\section{INTRODUCTION}

Elevated intracranial pressure (ICP) following acute brain injury may be due to cerebral edema, cerebral hyperperfusion, and/or intra- or extracranial hemorrhage. Intracranial hypertension (ICH) has been defined as elevated ICP $\geq 25 \mathrm{mmHg}$ for longer than 5 minutes in the absence of external noxious stimuli (1). ICH may be associated with secondary brain injury due to decreased cerebral perfusion pressure and cerebral ischemia.

Current intensive care unit (ICU) monitoring devices only allow for display of the ICP waveform with a digital parameter display of the averaged 3-10 second mean ICP value (2). ICP alarms are typically set for sustained periods of ICP in accordance with the definition of ICH as above. Thus, by the time a bedside ICP alarm goes off, the ICP may have been dangerously elevated for seconds to minutes, depending on the alarm settings and type of ICP elevation. Conversely, very brief elevations in ICP lasting less than 3-10 seconds may be missed altogether.

We have previously demonstrated that elevated ICP due to severe traumatic brain injury (TBI) is associated with changes in physiologic signal metrics derived from the electrocardiogram, arterial pressure waveform, and ICP waveform that suggests uncoupling of autonomic cardiovascular regulatory mechanisms (3-5). In addition, we reported preliminary data showing a breakdown in long-range correlation behaviour of heart rate fluctuations, as measured by the nonlinear scaling exponent, alpha $(\alpha)$, a nonlinear complexity statistic calculated by detrended fluctuation analysis $(6,7)$. This line of research suggests that metrics to analyze the ICP signal other than the time-averaged mean may have physiologic and clinical significance and that there are measurable differences in signal metrics between subjects with ICH.

In this study, the objective was to determine whether there were immediate changes in the ICP signal before, during, and after an acute elevations in ICP (a so-called "ICP spike") using metrics other than the time- 
averaged mean value. We hypothesized that measures of ICP complexity would decrease during periods of intracranial hypertension (ICH) compared to baseline values when the ICP was within physiologic normal ranges, providing evidence for decomplexification during an acute and finite period of severe physiologic stress (8). To test this hypothesis, we measured changes in the complexity of the ICP signal, estimated by the approximate entropy $(A p E n)$, as patients progressed from a stable state of normal ICP $(<25 \mathrm{mmHg})$ to intracranial hypertension (ICP $>25 \mathrm{mmHg}$ for longer than 5 minutes in the absence of external noxious stimuli (1), and then back towards pre-ICH levels.

Approximate entropy is a metric to estimate system complexity. A low value of $A p E n$ indicates predictability, regularity, or a quantitatively less complex state whereas high ApEn indicates unpredictability, irregularity, and greater complexity (9). ApEn is a computable measure which can be used to determine changing system complexity from time series and is potentially capable of classifying complex systems from a relatively small amount of data. It has been used mainly in the analysis of heart rate variability (10), endocrine hormone release pulsatility (11) and the impact of pulsatility on the ensemble orderliness of neurohormone secretion (12). Furthermore, ApEn has been applied to studies discriminating atypical EEGs (13) and respiratory patterns (14) from normative counterparts, to estimate depth of anesthesia (15) and to examine the time and frequency structure of Parkinson's disease tremor $(16,17)$. Preliminary evidence suggests that when applied to analysis of EEGs, the ApEn is predictive of epileptic seizure (18). ApEn has also been used to study the connection between panic disorder and respiration dynamics (19), to investigate changes during stages of consciousness, and to associate such alterations with brain function (20). This measure of complexity, however, has not been applied directly to the ICP signal in order to evaluate changes in system complexity during different pathologic states of brain injury. In this paper the term complexity is restricted to the scientific definition provided by Pincus as it relates to $A p E n(9)$. 


\section{MATERIALS AND METHODS}

\section{Patients and Patient Management and Data Acquisition}

The study protocol was reviewed and approved by the Institutional Review Board at Oregon Health \& Science University. The requirement for informed consent was waived.

This study included 11 ICP spikes from 7 subjects with brain injury admitted to the pediatric ICU at Doernbecher Children's Hospital. The subject's age, gender, mechanism and description of injury, admission Glasgow Coma Scale score (21), survival, and Glasgow Outcome Score (22) are listed in

Table 1. Management of the six subjects with severe traumatic brain injury (TBI) followed the recently published "Guidelines for the Acute Medical Management of Severe Traumatic Brain Injury in Infants, Children, and Adolescents" (1). One subject with a history of craniosynostosis and severe headaches was admitted for ICP monitoring.

\section{ICP Database and ICP Spike Detection}

Data for this study was obtained from the physiologic signal library in the Complex Systems Laboratory (2). The database consisted of 76 GB of ICP data collected from 93 patients from 1998-2003. ICP was monitored continuously using a ventricular catheter or parenchymal fiberoptic pressure transducer (Integra NeuroCare, Integra LifeSciences, Plainsboro, NJ). The ICP monitor was connected to a Philips Merlin patient monitor (Philips, Best, Netherlands) that sampled the ICP and arterial blood pressure signals at $125 \mathrm{~Hz}$. An HPUX workstation automatically acquired these signals through a serial data network, and they were stored in files on CD-ROM. Detailed description of the data acquisition system used in the Complex Systems Laboratory was previously reported (2). 
The following criteria were used to detect ICP spikes. The criteria are specified in terms of three nonoverlapping segments of the mean ICP signal: a 300 s stable region, a 10-300 s transition zone, and a $20 \mathrm{~s}$ region of ICH. Similar criteria were reported in an earlier study (23).

1. The difference between the minimum value in the critical region and the maximum value in the stable region was $\geq 10 \mathrm{mmHg}$. This ensured the detector only detected significant elevations of at least $10 \mathrm{mmHg}$ that occur over a period of no more than 5 minutes.

2. The minimum ICP value in the critical region was $>20 \mathrm{mmHg}$. This ensured that each ICP spike was large enough to be clinically significant (24).

3. The mean ICP was in the range of $0-100 \mathrm{mmHg}$ in the stable region and less than $150 \mathrm{mmHg}$ in the ICH region. These criteria were used to limit artifact from being detected as ICP spikes.

4. Each ICP spike was separated from preceding ICP spikes by at least 5 minutes. This ensured that a single long elevation in ICP was not detected as two separate ICP spikes.

To estimate the mean ICP, a low-pass filter was applied with a cutoff frequency sufficiently low to eliminate the pulsatile components of the ICP signal due to respiration and heart beats. The non-causal low-pass filter had a cutoff frequency of $0.22 \mathrm{~Hz}$ and zero phase delay. To decrease the computational load, the signal was decimated by a factor of 225 . This changed the effective sampling rate from $125 \mathrm{~Hz}$ to $0.556 \mathrm{~Hz}$. The criterion described in the previous section were then applied to every sample of the decimated signal to determine if a spike occurred.

ICP spikes that met the specified criteria were visually screened for artifact. This screen was based on a plot of the ICP signal spanning 20 minutes before and 30 minutes after the leading edge of the spike and a spectrogram of the same segment. The visual screen eliminated candidate spikes if (1) they contained artifact, (2) there was an abrupt drop in the ICP signal consistent with CSF withdrawal, (3) the signal was 
clipped, or (4) the ICP spike was part of the preceding episode of intracranial hypertension. If there were no problems detected during the 50 minute record, the ICP spike was included in the study.

The automatic spike detection algorithm found 166 ICP segments that met our criteria for an acute spike. During the visual screen we found that 31 of the segments contained artifact, 28 of which were actually periods of cerebral spinal fluid drainage when the ventriculostomy catheter was turned "off" to the pressure monitor and created a "false" spike. An additional 95 segments were "clipped" at the maximum range of the patient monitor (i.e. the top or bottom of the ICP waveform was cut off creating signal artifact not suitable for analysis - this technical problem has since been resolved). One segment was identified as a second detection of a single ICH episode. The end result was 11 clean records of ICH detected from 7 different subjects that were used for analysis. An example of a detected ICH region is shown in Figure 1. Figure 2 shows an example of the ICP segments used for analysis including the stable, ICH and recovering regions.

\section{Approximate Entropy}

Approximate Entropy $(A p E n)$ is a family of parameters and statistics introduced as a quantification of regularity in time-series, initially motivated by applications to short and noisy data sets. It was first proposed by Pincus in 1991 (9), and its biologic and physiologic applications are spreading rapidly (25). Several properties of ApEn facilitate its utility for empirical time series analysis (26):

1. ApEn is nearly unaffected by noise below a de facto specified filter level $(r)$.

2. ApEn can be applied to time series of 50 or more points with good reproducibility.

3. ApEn is finite for stochastic, noisy deterministic and composite processes.

4. Increasing values of $A p E n$ correspond to more irregularity in the time series or to intuitively increasing process complexity. 
The potential uses of $A p E n$ to provide new insights in epidemiologic settings are considerable from a complementary perspective to that given by more classical statistical methods. It appears that $A p E n$ has potential widespread utility to practical data analysis and clinical application due to the salient features it bears. Moreover, when applied to the analysis of biomedical time series, ApEn does not show the important drawbacks - i.e. very long data sequences needed to estimate them accurately, data must be stationary, etc. - that many widely-applied nonlinear methods (correlation dimension, first positive Lyapunov exponent) have.

$A p E n$ is scale invariant, model independent, evaluates both dominant and subordinant patterns in data, and discriminates series for which clear feature recognition is difficult; notably it detects changes in underlying episodic behaviour not reflected in peak occurrences or amplitudes (27). ApEn assigns a nonnegative number to a time series, with larger values corresponding to more complexity or irregularity in the data. It has two user-specified parameters: a run length $m$ and a tolerance window $r$. Briefly, ApEn measures the logarithmic likelihood that runs of patterns that are close (within $r$ ) for $m$ contiguous observations remain close (within the same tolerance width $r$ ) on subsequent incremental comparisons. $A p E n$ has two user-specified parameters: a run length $m$ and a tolerance window $r$. It is important to consider $\operatorname{ApEn}(m, r)$ - or $\operatorname{ApEn}(m, r, N)$, where $N$ is the number of points of the time series - as a family of parameters: comparisons between time series segments can only be made with the same values of $m$ and $r(26)$.

Formally, given $N$ data points from a time series $<x(n)>=x(1), x(2), \ldots, x(N)$, to compute $A p E n$, the following steps are taken:

1. Form $m$-vectors $X(1) \sim X(N-m+1)$ defined by: $X(i)=[x(i), x(i+1), \ldots, x(i+m-1)], i=1 \sim N-m+1$. These vectors represent $m$ consecutive $x$ values, commencing with the $i$ th point.

2. Define the distance between $X(i)$ and $X(j), d[X(i), X(j)]$, as the maximum absolute difference 
between their respective scalar components:

$$
d[X(i), X(j)]=\max _{k=1,2, \ldots, m}(|x(i+k-1)-x(j+k-1)|)
$$

3. For a given $X(i)$, count the number of $\mathrm{j}(j=1 \sim N-m+1, j \neq i)$ such that $d[X(i), X(j)] \varangle r$, denoted as $N^{m}(i)$. Then, for $i=1 \sim N-m+1$,

$$
C_{r}^{m}(i)=N^{m}(i) /(N-m+1)
$$

The $C_{r}^{m}(i)$ values measure within a tolerance $r$ the regularity, or frequency, of patterns similar to a given one of window length $m$.

4. Compute the natural logarithm of each $C_{r}^{m}(i)$, and average it over $i$,

$$
\phi^{m}(r)=\frac{1}{N-m+1} \sum_{i=1}^{N-m+1} \ln C_{r}^{m}(i)
$$

$\phi^{m}(r)$ represents the average frequency that all the $m$-point patterns in the sequence remain close to each other.

5. Increase the dimension to $m+1$. Repeat steps 1 to 4 and find $C_{r}^{m+1}(i)$ and $\phi^{m+1}(r)$.

6. Approximate Entropy is then defined by:

$$
\operatorname{ApEn}(m, r, N)=\phi^{m}(r)-\phi^{m+1}(r)
$$

Prior to ApEn estimation, the ICP signals were filtered to eliminate the low frequency components (baseline trend) and remove the mean pressure (DC component). We used a high-pass equiripple FIR filter with a cut-off frequency of $0.5 \mathrm{~Hz}$. This guaranteed that our ApEn estimates obtained from the ICP signal were based exclusively on the ICP pulse morphology, since both the mean ICP and the baseline trend were eliminated with by the highpass filter. Figure 2 and Figure 3 illustrate this methodology. Each filtered ICP signal was windowed into segments of 20 seconds in duration. ApEn was estimated for each segment. We used normalized parameters of $m=1$ and $r=20 \%$ of the segments time series standard deviation (SD) (12). Normalizing $r$ in this manner gives $A p E n$ a translation and scale invariance, in that it 
remains unchanged under uniform process magnification, reduction, or constant shift to higher or lower values $(26)$. Several studies $(9,27,28)$ have demonstrated that these input parameters produce good statistical reproducibility for $A p E n$ for time series of length $n \geq 60$, as considered herein.

\section{Statistical Analysis}

Statistical analysis was aimed at determining the statistical significance of the mean reduction in $A p E n$ during the ICH period for each of the individual subjects and across the population. Mean ApEn's values were obtained for 2 min windows immediately before, during, and after the ICH period. We used bootstrap to estimate the standard error of the means for each of these states. We performed a nonparametric hypothesis test in order to determine statistically significant mean ApEn reductions based on bootstap (29). The main advantage of using the nonparametric bootstrap technique is that is that it can be used to assess the statistical significance of these reductions without making any assumptions about the distribution of the mean $A p E n$ reductions. The nonparametric bootstrap hypothesis testing involves computing a bootstrap confidence interval for the difference of mean $A p E n$. Equality of the mean $A p E n$ is obtained if zero is a possible value in the confidence interval. Results were considered to be statistically significant if $\mathrm{p}<0.01$.

\section{RESULTS}

Table 2 shows the estimated mean $A p E n$ during the stable, ICH, and recovering regions for each of the 11 episodes. Table 3 shows the estimated standard errors corresponding to the ApEn means shown in Table

2. The mean $A p E n$ was lower during the ICH period than during the stable and recovering period in all the 11 episodes $(\mathrm{p}<0.01)$ (Table 2$)$. The population mean across all the 11 episodes during the stable, $\mathrm{ICH}$, and recovering regions were estimated to be $0.5158 \pm 0.0089,0.3887 \pm 0.077$, and $0.5096 \pm 0.0158$, respectively. Both the mean reduction in $A p E n$ from the state of normal ICP (stable region) to the ICH region (-0.1271), and the increase in $A p E n$ from the ICH region to the recovering region (0.1209) were 
determined to be statistically significant $(\mathrm{p}<0.01)$. Figure 4 shows histograms of the mean ApEn for the stable, ICH, and recovering regions. Figure 5 shows a plot of the normalized ApEn for each of the 11 episodes and the median ApEn across all the episodes. The estimated ApEn decreases as subjects progressed from a stable state of normal ICP to ICH. The level of complexity begins to return within minutes as ICP drops below 20-25 mmHg. Figure 6a-b shows the ApEn vs. ICP for two representative subjects during the study period.

\section{DISCUSSION}

Our main finding was that $A p E n$ of the ICP signal decreased as patients progressed from a state of normal ICP to a state of ICH. As larger $A p E n$ values correspond to increased complexity or irregularity in the data, this indicates that decreased complexity of ICP coincides with episodes of ICH. We also noted that the level of complexity begins to increase and return towards baseline levels within minutes as ICP drops below $20-25 \mathrm{mmHg}$. These findings suggest that the complex regulatory mechanisms that govern ICP are disrupted during acute rises in ICP and return towards baseline with resolution of the acute elevation in pressure. Although we only analyzed one subject without severe TBI as a cause of ICH, the results were similar in this subject suggesting that the regulatory mechanisms that govern ICP are independent of the type of injury.

\section{Physiologic Implications}

Godin and Buchman have suggested that the pathogenesis of multiple organ dysfunction syndrome (MODS) from the systemic inflammatory response syndrome (SIRS) may be due to erosion of interconnections among organ systems (30). Loss of variability in the heart beat and systemic blood pressure has been demonstrated during the sepsis syndrome in experimental models and septic patients (31-33). Return of variability was reported by Ellenby et al. to be associated with recoupling between the 
autonomic nervous and cardiovascular systems during septic shock (34). Our results suggest that analogous physiologic mechanisms occur during ICH, at least during short periods of observation.

Zwiener et al. (35) found evidence of impaired short-term (25-60 second periods) dynamics between respiratory movements and fluctuations in heart rate and blood pressure brain injured subjects compared to controls. Furthermore, the impairment in short-term dynamics were inversely proportional to the degree of neurological injury and were not affected by concomitant analgesic or sedative medications. They concluded that the diminished coherence was indicative of organ system uncoupling or decomplexification consistent with previous reports $(8,30)$.

Zweiner et al. further suggest that the short-term dynamics of coherence and coordination of multiple organ system activity depends on brainstem afferent activities (35). They point to research by Langhorst et al. (36) and Schulz et al. (37) who found that the degree and pattern of rhythmic fluctuations in brainstem neurons were related to respiratory or cardiovascular functions and the joint influence on the efferent neuroautonomic activity to lung, heart, and blood vessels depended on brainstem afferent activity. As the main stimuli to the brainstem in these experiments were from peripheral somatic and visceral origins, the conclusion is that these experiments were an example of autonomic adaptations to changed external conditions, a postulated rationale for the advantages of complexity in healthy organisms $(30,35)$. Thus, uncoupling between lungs, heart, and the vascular system decreases their functional performances and results in worse outcome.

Zweiner at al. (35) also point out, and we agree, that it is not clear whether our findings of decomplexification represents a primary physiological process or is only an epiphenomenon. However, the results of the current study, using more sensitive nonlinear methodologies, strongly suggest the former. 
The six subjects with severe TBI had an evolving clinical course. It is clinically accepted that maximal ICP generally occurs 24 to 48 hours following severe TBI. If the SIRS to MODS analogy applies in TBI, then future studies need to examine if repeated episodes of decreased complexity in ICP, indicative of loss of regulatory control mechanisms, result in worsening brain injury, cerebral edema, neuronal cell death, and, in extreme cases, eventually lead to brain death. In addition, there has been much discussion about what is the best treatment threshold value of ICP to use in severe TBI $(1,38)$. Loss of physiologic complexity of the ICP signal may be a sensitive and specific method for determination of exactly at what value elevated ICP becomes physiologically dangerous.

\section{Clinical Implications - Measures of Cerebral Autoregulation}

Investigators have suggested that diminished complexity results in a decreased ability to respond to external perturbations $(30,39)$. Under normal conditions, cerebral autoregulatory mechanisms maintain cerebral blood flow to the brain constant over a wide range of systemic arterial pressures with typical values between 45 and $65 \mathrm{ml} / 100 \mathrm{mg}$ of brain tissue per second despite variations in blood pressure as large as $100 \mathrm{mmHg}(40)$. Local cerebral autoregulation delivers a relatively constant cerebral perfusion pressure in response to fluctuations in ICP such as with postural change and cough.

It is clear that acute ICH is a result of failure of normal cerebral autoregulatory mechanisms to compensate for overwhelming changes in cerebral volume (hemorrhage or edema), external pressure (depressed skull fractures), cerebral blood volume (cerebral hyperperfusion), or cerebral spinal fluid (obstructive hydrocephalus). Our findings suggest that, similar to sepsis, the ICP waveform exhibits diminished complexity and increased regularity during periods of ICH when CAR has failed. Conversely, when CAR is intact, the ICP becomes more complex and irregular. Thus, our findings suggest that $A p E n$ of the ICP waveform may provide an indirect measure of CAR. 
Other investigators have proposed a number of indirect measures to determine whether CAR is intact or not. Physiologic stressors or challenges such as changes in $\mathrm{PaO}_{2}, \mathrm{PaCO}_{2}, \mathrm{ABP}$, intracranial volume (via balloon catheter) have all been reported to correlate to various degrees with CAR. These techniques require manipulation of one or more physiologic variables with some inherent risk to the patient. More recently, investigators have examined the transfer function and phase shift between cerebral blood flow and ICP as a dynamic measure of CAR (41-43). These measures typically use Doppler blood flow velocity in the middle cerebral artery that may be difficult to maintain for continuous measurements in the ICU environment. Direct analysis of the ICP waveform using ApEn or some other nonlinear metric may provide an easier and less risky alternative for determination of CAR. Currently there are a few indices of potential clinical interest obtained by direct analysis of the ICP signal (e.g. RAP, pressure-reactivity). The RAP index (index of compensatory reserve) is defined as the correlation coefficient (R) between the ICP pulse amplitude (AMP) and mean pressure (P). This index is obtained by calculating the linear correlation between consecutive, time average data points of AMP and ICP (usually about 40 of such samples are used), and indicates the degree of correlation between AMP and mean ICP over short periods of time ( 4 $\min )$. This can be used to estimate the state of the pressure-volume compensatory reserve. Another ICP derived index is the pressure-reactivity index. This index has been shown to correlate well with indices of autoregulation based on transcranial Doppler ultrasonography. In conjunction these indices can be use to indirectly estimate the CAR or deranged cerebrospinal compensatory reserve (44)

\section{Interpretation of Approximate Entropy}

Since it is not possible to directly measure the complexity of individual organ systems, approximate measures must be used. There are several methods to estimate the complexity of systems from analysis of time series. In this work the meaning of the term complexity is restricted to the definition provided by Pincus in relation to the $A p E n$ (9). We chose to used $A p E n$ because it was specifically designed as a 
technique to determine changing system complexity from short time series. Furthermore, contrary to the frequent used correlation dimension measure of complexity, ApEn does not assume an underlying deterministic model or chaos and is capable of classifying complex systems that include chaotic, stochastic and composite processes; these last processes are likely models for complicated biological data sets. In the general stochastic, noisy deterministic or composite setting, the statistical accuracy of the correlation dimension measure of complexity is typically very poor $(9,45)$. Because dynamic mechanics of most biological signals remain undefined, a suitable statistic of complexity for these signals must be more cautious to accommodate general classes of processes and their much more diffuse reconstructed dynamics (26). Moreover, a robust estimate of $A p E n$ can be obtained by using a number of points several orders of magnitude lower than that needed to estimate accurately the correlation dimension or the first positive Lyapunov exponent (25).

In order to aid the interpretation of the $A p E n$ values obtained in this study, we performed a series of test with synthetic signals with known characteristics. From these test we concluded that 1) the ApEn increases as the frequency and the number of harmonics of a sinusoidal signal increases, 2) ApEn is correlated with noise bandwidth, increasing as the noise bandwidth increases ( $A p E n$ is lower in the case of colored noise than for white noise), 3) typical values of $A p E n$ for sinusoidal signals range from 0.001 to 0.07 (increasing as the numbers of harmonics increases) and 1.4 to 2 for white noise, and 4) the $A p E n$ of ICP ranges from 0.05 to 1.5 , correlating with heart rate variability, noise power, and pulse morphology changes.

The fact that $A p E n$ correlates with the number of harmonics in periodic and quasi-periodic signals has important implications in the context of ICP analysis, since the number of harmonics of the signal directly relates to the morphology of the ICP beats. Investigators have documented specific variations in the ICP beat morphology, which correspond to specific alterations in the cerebral vascular system, CSF 
circulation, and respiration. These morphology variations may be used to measure the progression of disease. For instance, Pornoy states that in patients who do not have a cerebral edema or expanding mass, the ICP beat shows an initial sharp rise and subsequent downward slope similar to the arterial pulse; but as the expanding mass or edema develops, the ICP pulse becomes more rounded (46). As the ICP pulse becomes more rounded the amplitude of the higher frequency sinusoidal components also decreases.

In the light of our results $A p E n$ can be interpreted as a "summarizing metric" that combines information such as heart rate variability, number of harmonics, and time morphology variability. Our results support the hypothesis that $A p E n$ is inversely related and negatively correlated to acute elevations in ICP. Furthermore, our results also support the hypothesis that there is information in the ICP pulse which correlates with severity of disease, since $A p E n$ was calculated from high-pass filtered ICP signals where the mean ICP trend was removed prior the the ApEn calculation.

\section{Limitations}

The data presented in this study came from only seven subjects. Further study is warranted in a larger and more diverse population of subjects with ICH from a variety of diseases and injuries. Additionally, the there was no direct or indirect measure of cerebral autoregulation during periods of ICH. Finally, the dataset was blinded to treatment(s) used in response to ICH so it is not possible to differentiate what effects, if any, therapies such as osmotic diuresis, cerebral spinal fluid drainage, mild hyperventilation, or elevation of the head-of-the bed may have had on ICP analysis. 


\section{ACKNOWLEDGEMENT}

The authors would like to thank Matthew Goldstein for his help in the preparation of this manuscript. 


\section{REFERENCES}

1. Adelson PD, Bratton S, Carney N, Chesnut R, du Cordray H, Goldstein B, Kochanek P, Miller H, Partington M, Selden N, Warden C, Wright D. Guidelines for the Acute Medical Management of Traumatic Brain Injury in Infants, Children and Adolescents. Crit Care Med 2003:31:S417-S490.

2. Goldstein B, McNames J, Ellenby M, Ibsen L, Jacques S, Aboy M, Thong T, Phillips C, Levitte G. ICU Monitoring of Physiologic Signals - Engineering Aspects, Clinical Interpretation, and Future Directions. In: Current Concepts in Pediatric Critical Care Course. Shanley TP (Ed). Soc Crit Care Med, Des Planes, IL. 2004:201-229.

3. Goldstein B, DeKing DE, DeLong D, et al. The autonomic cardiovascular state following severe brain injury and brain death. Crit Care Med 1993;21:228-233.

4. Goldstein B, Kempski MH, Tipton RB, et al. Heart rate power spectral and plasma catecholamine changes following acute brain injury in children. Crit Care Med 1996;24:234-240.

5. Goldstein B, Toweill D, Lai S, Sonnenthal K, Kimberly B. Uncoupling of the autonomic and cardiovascular systems in acute brain injury. Am J Physiol - Regulatory, Integrative and Comparative Physiol 1998;44:R1287-1292.

6. Peng CK, Havlin S, Stanley HE, Goldberger AL. Quantification of scaling exponents and crossover phenomena in nonstationary heartbeat time series. Chaos 1995;5:82-87

7. Goldstein B, Peng CK, Sonnenthal K, Kimberly B, Goldberger AL. Detrended fluctuation analysis in acute brain injury: Evidence for breakdown of long-range correlation behavior in beat-to-beat fluctuations of heart rate (abstract). Pediatrics 1996:98:550

8. Goldstein B, Fiser D, Kelly MM, Mickelsen DM, Ruttimann U, Pollack MM. Decomplexification in critical illness and injury: The relationship between heart rate variability, severity of illness, and outcome. Crit Care Med 1998:26;352-357.

9. Pincus SM. Approximate entropy as a measure of system complexity. Proceedings of the National Academy of Sciences of the USA 1991; 88: 2297-2301. 
10. Pincus SM, Goldberger AL. Physiological time series analysis: what does regularity quantify? American Journal of Physiology: Heart and Circulatory Physiology 1994; 266: H1643-H1656.

11. Pincus SM. Older males secrete luteinizing hormone and testosterone more irregularly and joint more asynchronously, than younger males. Proceedings of the National Academy of Sciences of the USA 1996; 93: 14100-14105.

12. Veldhuis JD, Johnson ML, Veldhuis OL, Straume M, Pincus SM. Impact of pulsatility on the ensemble orderliness (approximate entropy) of neurohormone secretion. American Journal of Physiology: Regulatory, Integrative and Comparative Physiology 2001; 281: 1975-1985.

13. Bruhn J, Röpcke H, Rehberg B, Bouillon T, Hoeft A. Electroencephalogram approximate entropy correctly classifies the occurrence of burst suppression pattern as increasing anesthetic drug effect. Anesthesiology 2000;93:981-985.

14. Engoren M. Approximate entropy of respiratory rate and tidal volume during weaning from mechanical ventillation. Critical Care Medicine 1998;26:1817-1823.

15. Zhang X-S, Roy RJ. Derived fuzzy knowledge model for estimating the depth of anesthesia. IEEE Transactions on Biomedical Engineering 2001; 48 (3): 312-323.

16. Vaillancourt DE, Newell KM. The dynamics of resting and postural tremor in Parkinson's disease. Clin Neurophysiol 2000;111:2046-2056.

17. Vaillancourt DE, Slifkin AB, Newell KM. Regularity of force tremor in Parkinson's disease. Clin Neurophysiol 2001;112:1594-1603.

18. Radhakrishnan N, Gangadhar BN. Estimating regularity in epileptic seizure time-series data. A complexity-measure approach. IEEE Engineering in Medicine and Biology 1998; 17 (3): 89-94.

19. Caldirola D, Bellodi L, Caumo A, Migliarese G, Perna G. Approximate Entropy of Respiratory Patterns in Panic Disorder. Am. J. Psychiatry 2004, 161, pp. 79-87.

20. Burioka N, Cornéliessen G, Halberg F, Kaplan DT, Suyama H, Sako T, Shimizu E. Approximate Entropy of Human Respiratory Movement During Eye-Closed Waking and Different Sleep Stages. Chest 
2003, 123, pp. 80-86.

21. Teasdale G, Jennett B. Assessment of coma and impaired consciousness. A practical scale. Lancet $1974 ; 2: 81-84$

22. Jennett B, Bond M. Assessment of outcome after severe brain damage. Lancet 1975;480-484.

23. McNames J, Crespo C, Bassale J, Aboy M, Ellenby M, Lai S, Goldstein B. Sensitive precursors to acute episodes of intracranial hypertension. Proceedings of the 4th International Workshop in Biosignal Interpretation. 2002:303-306.

24. Luerssen G. Intracranial Pressure: Current Status in Monitoring and Management. Seimnars in Pediatric Neurology 1997;4:146-155.

25. Fusheng Y, Bo H, Qingyu T. Approximate entropy and its application in biosignal analysis. In: Akay M, editor. Nonlinear Biomedical Signal Processing Volume II: Dynamic Analysis and Modeling. New York: IEEE Press Series on Biomedical Engineering, 2001:72-91.

26. Pincus SM. Assessing serial irregularity and its implications for health. Annals of the New York Academy of Sciences 2001; 954: 245-267.

27. Pincus SM, Keefe DL. Quantification of hormone pulsatility via an approximate entropy algorithm. American Journal of Physiology 1992; 262: E741-E754.

28. Kaplan DT, Furman MI, Pincus SM, Ryan SM, Lipsitz LA, Goldberger AL. Aging and the complexity of cardiovascular dynamics. Biophysical Journal 1991; 59: 945-949.

29. Efron B, Tibshirani RJ. An Introduction to Bootstrap. Chapman \& Hall/CRC. 1994

30. Godin PJ, Buchman TG. Uncoupling of biological oscillators: A complementary hypothesis concerning the pathogenesis of multiple organ dysfunction syndrome. Crit Care Med 1996:24:1107-1116. 31. Goldstein B, Kempski MH, Stair DB, Tipton RB, DeKing DE, DeLong DJ, Cox C, Lund N, Woolf PD. Autonomic modulation of heart rate variability during endotoxin shock in rabbits. Crit Care Med $1995 ; 23 ; 1694-1702$. 
32. Garrard CS, Kontoyannis DA, Piepoli M. Spectral analysis of heart rate variability in the sepsis syndrome. Clinical Autonomic Research. 1993;3:5-13.

33. Piepoli M, Garrard CS, Kontoyannis DA, Bernardi L. Autonomic control of the heart and peripheral vessels in human septic shock. Intensive Care Medicine. 1995;21:112-119.

34. Ellenby MS, McNames J, Lai S, McDonald BA, Krieger D, Sclabassi RJ, Goldstein B Uncoupling and recoupling of autonomic regulation of the heart beat in pediatric septic shock. Shock. 2001; 16:274-7.

35. Zwiener U, Schelenz Ch, Bramer S, Hoyer D. Short-term dynamics of coherence between respiratory movements, heart rate, and arterial pressure fluctuations in severe acute brain disorders. Physiol Res. $2003 ; 52: 517-524$.

36. Langhorst P, Schulz B, Schulz G, Lambertz M. Reticular formation of the lower brain stem. A common system for cardiorespiratory and somatomotor functions: discharge patterns of neighbouring neurons influenced by cardiorespiratory and respiratory afferents. J Auton Nerv Syst 1983;9:411-432. 37. Schulz G, Lambertz M, Schulz B, Langhorst P, Krienke B. Reticular formation of the lower brain stem. A common system for cardiorespiratory and somatomotor functions. Discharge patterns of neighbouring neurons influenced by cardiorespiratory and respiratory afferents. J Auton Nerv Syst $1985 ; 12: 35-62$.

38. Bullock R, Chesnut RM, Clifton G, et al: Guidelines for the management of severe traumatic brain injury. J Neurotrauma 2000; 17:451-553.

39. Tjardes T, Neugebauer E. Sepsis research in the next millennium: concentrate on the software rather than the hardware. Shock. 2002;17:1-8.

PMID: 11795662 [PubMed - indexed fo

40. Latka M, Was Z, Kolodziej W, Latka D, Goldstein B, West BJ. Phase synchronization in cerebral hemodynamics. Physical Review Letters (under review). 
41. Piper IR, Miller JD, Dearden NM, Leggate JR, Robertson I. Systems analysis of cerebrovascular pressure transmission: an observational study in head-injured patients. J Neurosurg. 1990 Dec;73(6):87180.

42. Zhang R, Zuckerman JH, Giller CA, Levine BD. Transfer function analysis of dynamic cerebral autoregulation in humans. Am J Physiol - Heart Circ Physiol 1998;274:H233-H241.

43. ter Minassian A, Dube L, Guilleux AM, Wehrmann N, Ursino M, Beydon L. Changes in intracranial pressure and cerebral autoregulation in patients with severe traumatic brain injury. Crit Care Med 2002;30:1616-1622

44. Czosnyka M, Pickard JD. Monitoring and interpretation of intracranial pressure. J. Neurol Neurosurg Psychiatry 2004;75:813-821

45. Pincus SM, Singer BH. Randomness and degrees of irregularity. Proc Natl Acad Sci USA 1996;93:2083-2088.

46. Pornoy HD, Choop M. Spectral Analsysis of intracranial pressure. In: Shulman K, et al, eds. Intracranial Pressure IV. New York: Springer-Verlag, 1980:167-172. 
Table 1. Subject characteristics.

\begin{tabular}{|c|c|c|c|c|c|c|}
\hline Subject & Age & Gender & $\begin{array}{l}\text { Mechanism and description of } \\
\text { brain injury }\end{array}$ & $\begin{array}{l}\text { Admission } \\
\quad \text { GCS }\end{array}$ & Survival & GOS \\
\hline 1 & 9.5 & $\mathrm{~F}$ & $\begin{array}{l}\text { Fall off horse; } \\
\text { Depressed skull fracture, IPH, } \\
\text { cerebral edema }\end{array}$ & 7 & $\mathrm{Y}$ & 4 \\
\hline 2 & 4.5 & M & $\begin{array}{l}\text { MVA; } \\
\text { Skull fracture, SDH, cerebral edema }\end{array}$ & 3 & $\mathrm{Y}$ & 3 \\
\hline 3 & 8 & $\mathrm{~F}$ & $\begin{array}{l}\mathrm{s} / \mathrm{p} \text { craniosysostosis repair at age } 2 \\
\text { y.o., ICP monitoring for headaches }\end{array}$ & 15 & $\mathrm{Y}$ & NA \\
\hline 4 & 4.75 & $\mathrm{~F}$ & $\begin{array}{l}\text { MVA; } \\
\text { Skull fracture, IPH, cerebral edema }\end{array}$ & 5 & $\mathrm{Y}$ & 2 \\
\hline 5 & 11.5 & M & $\begin{array}{l}\text { MVA; } \\
\text { Skull fracture, SDH }\end{array}$ & 8 & $\mathrm{Y}$ & 3 \\
\hline 6 & 12.5 & M & $\begin{array}{l}\text { Gun Shot Wound; } \\
\text { IPH, SDH, cerebral edema }\end{array}$ & 4 & Y & 4 \\
\hline 7 & 15.8 & M & $\begin{array}{l}\text { Fall off skateboard; } \\
\text { Depressed skull fracture, IPH, } \\
\text { cerebral edema }\end{array}$ & 3 & $\mathrm{Y}$ & 3 \\
\hline Mean \pm SD & $9.5 \pm$ & & & $6 \pm 4$ & & $3 \pm 1$ \\
\hline
\end{tabular}

GCS, Glasgow Coma Scale score GOS, Glasgow Outcome Score IPH, intraparenchymal hematoma MVA, motor vehicle accident SDH, subdural hematoma 
Table 2. Mean ApEn of the intracranial pressure signal for the stable, intracranial hypertension, and recovering regions. Standard errors for each of the columns are shown in Table 3.

\begin{tabular}{lll}
\hline Stable & Critical & Recovering \\
\hline 0.643 & 0.262 & 0.759 \\
0.536 & 0.325 & 0.446 \\
0.548 & 0.387 & 0.539 \\
0.439 & 0.353 & 0.433 \\
0.499 & 0.336 & 0.377 \\
0.605 & 0.225 & 0.442 \\
0.507 & 0.363 & 0.466 \\
0.233 & 0.223 & 0.234 \\
0.329 & 0.225 & 0.325 \\
0.341 & 0.197 & 0.326 \\
0.315 & 0.185 & 0.277 \\
0.315 & 0.185 & 0.277 \\
\hline
\end{tabular}


Table 3. Standard error of the mean $A p E n$ for the stable, intracranial hypertension, and recovering regions.

\begin{tabular}{lll}
\hline Stable & Critical & Recovering \\
\hline 0.0157 & 0.0209 & 0.0070 \\
0.0060 & 0.0358 & 0.0153 \\
0.0066 & 0.0032 & 0.0122 \\
0.0037 & 0.0026 & 0.0095 \\
0.0048 & 0.0069 & 0.0014 \\
0.0142 & 0.0078 & 0.0094 \\
0.0028 & 0.0024 & 0.0035 \\
0.0006 & 0.0004 & 0.0005 \\
0.0023 & 0.0015 & 0.0174 \\
0.0060 & 0.0032 & 0.0078 \\
0.0058 & 0.0023 & 0.0047 \\
0.0058 & 0.0023 & 0.0047 \\
\hline
\end{tabular}




\section{LEGENDS FOR FIGURES}

Figure 1. Illustration of the criteria for an acute elevation in intracranial pressure. The signal divided into three segments: a 5 min stable region (SR), a 10-300 s transition zone (TZ), and a $20 \mathrm{~s}$ critical region (CR). The stable and critical regions had a required separation of at least $10 \mathrm{mmHg}$.

Figure 2. Illustration of the type of intracranial pressure (ICP) spike analysed in this study. The light grey is the ICP signal waveform sampled at $125 \mathrm{~Hz}$, and the dark line is the moving average ICP pressure. All ICP segments studied were 50 minutes long, with the onset of the ICH period synchronized at minute 20.

Figure 3. Prior to ApEn estimation, the intracranial pressure (ICP) signals were filtered to eliminate the low frequency components (baseline trend) and remove the mean pressure (DC component). This figure illustrates the results of this operation. Processing the high passed ICP signal ensures that the ApEn metric obtained is based entirely on the ICP beat morphology, since the ICP mean pressure information is eliminated in this operation.

Figure 4. Histogram approximating the sampling distribution of the mean $A p E n$ across all the patients for the stable, critical (ICH), and recovering regions obtained using bootstrap. Note the statistically significance reduction in mean $A p E n$ during the ICH period.

Figure 5. ApEn (normalized) for each of intracranial hypertension (ICH) episode (light grey) and mean and median across all ICH episodes (dark). ApEn decreases as patients progressed from a stable state of normal ICP to a state of ICH. This indicates that decreased complexity of ICP coincides with episodes of ICH in TBI

Figure 6.a. ApEn vs. ICP for Patient 1, episode 1. Note decreased $A p E n$ associated with both ICP spikes in pressure $>20 \mathrm{mmHg}$. 6.b. ApEn vs. ICP for Patient 3, episode 1. Note drop in ApEn during ICP spikes with return to baseline levels during normal ICP. 
Figure 1.

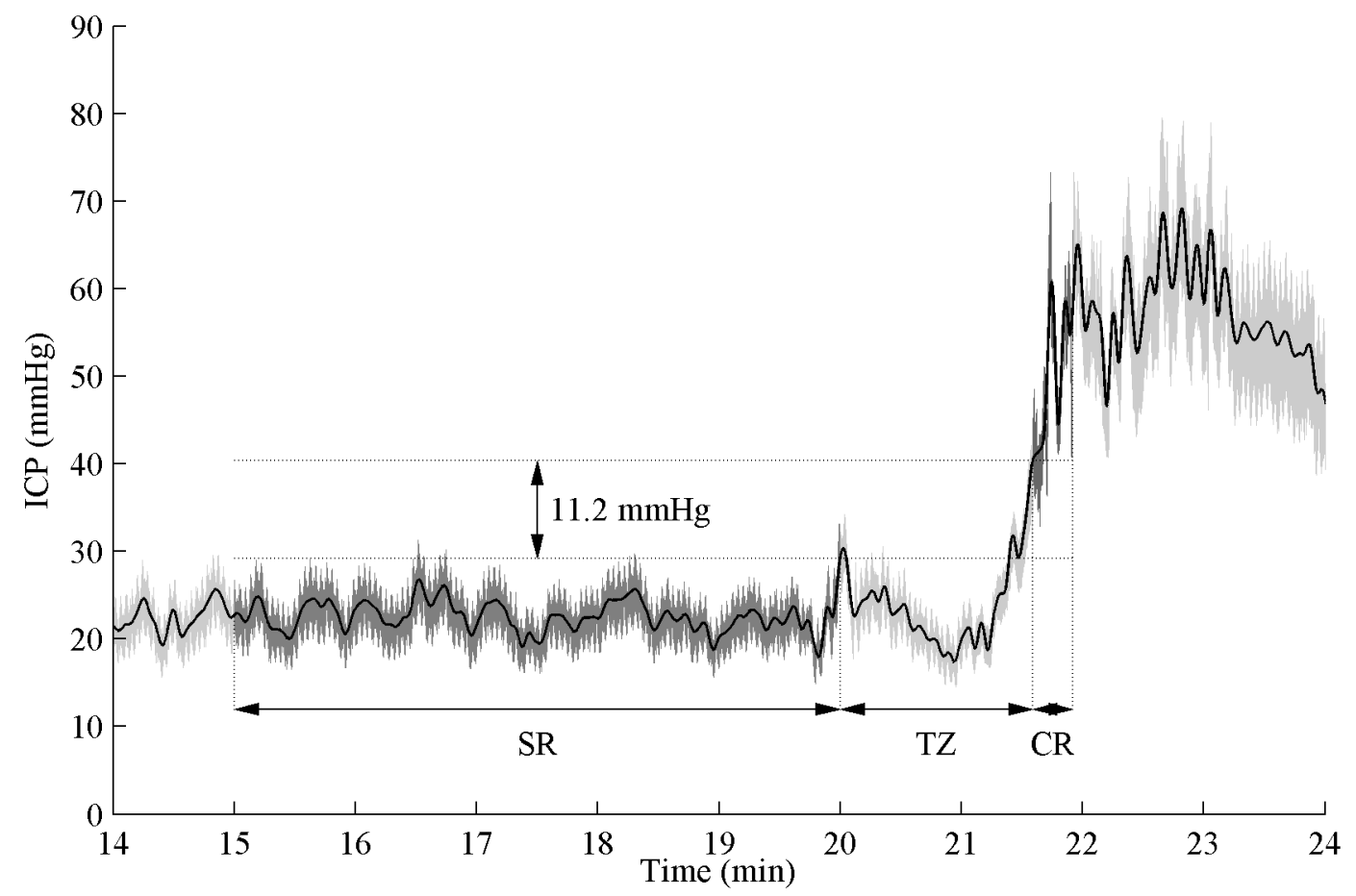

Figure 2.

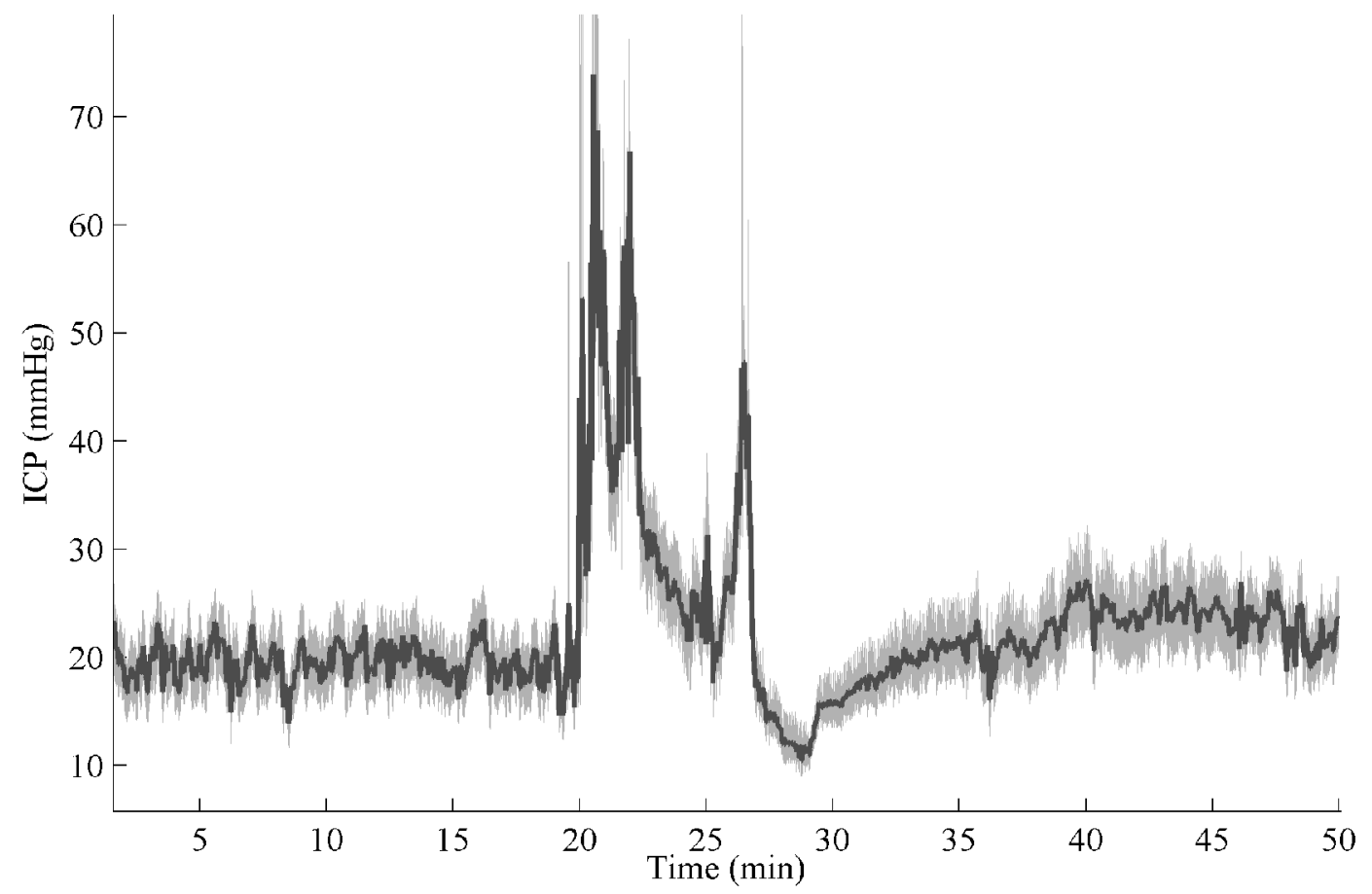


Figure 3.

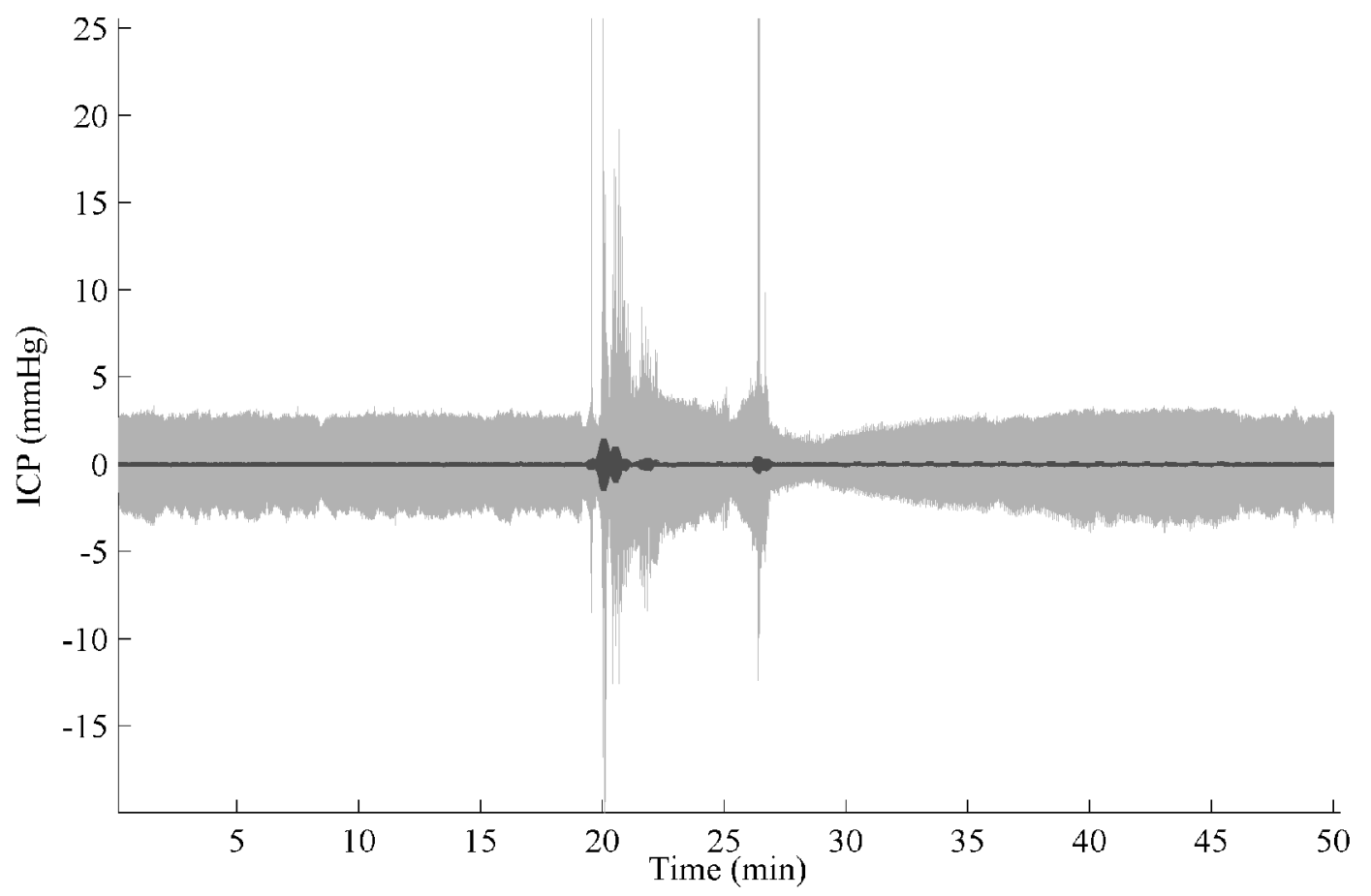

Figure 4.

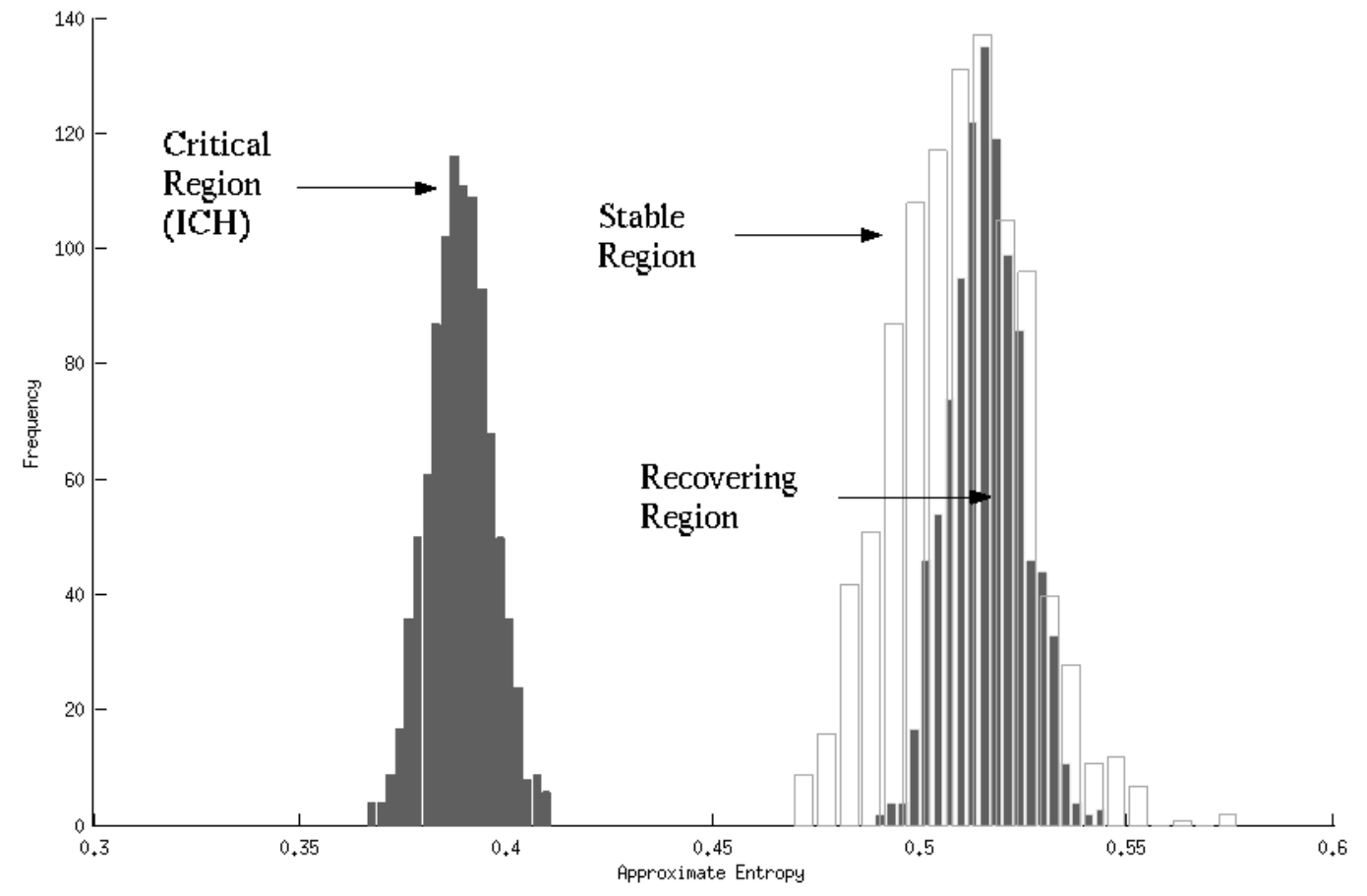


Figure 5.

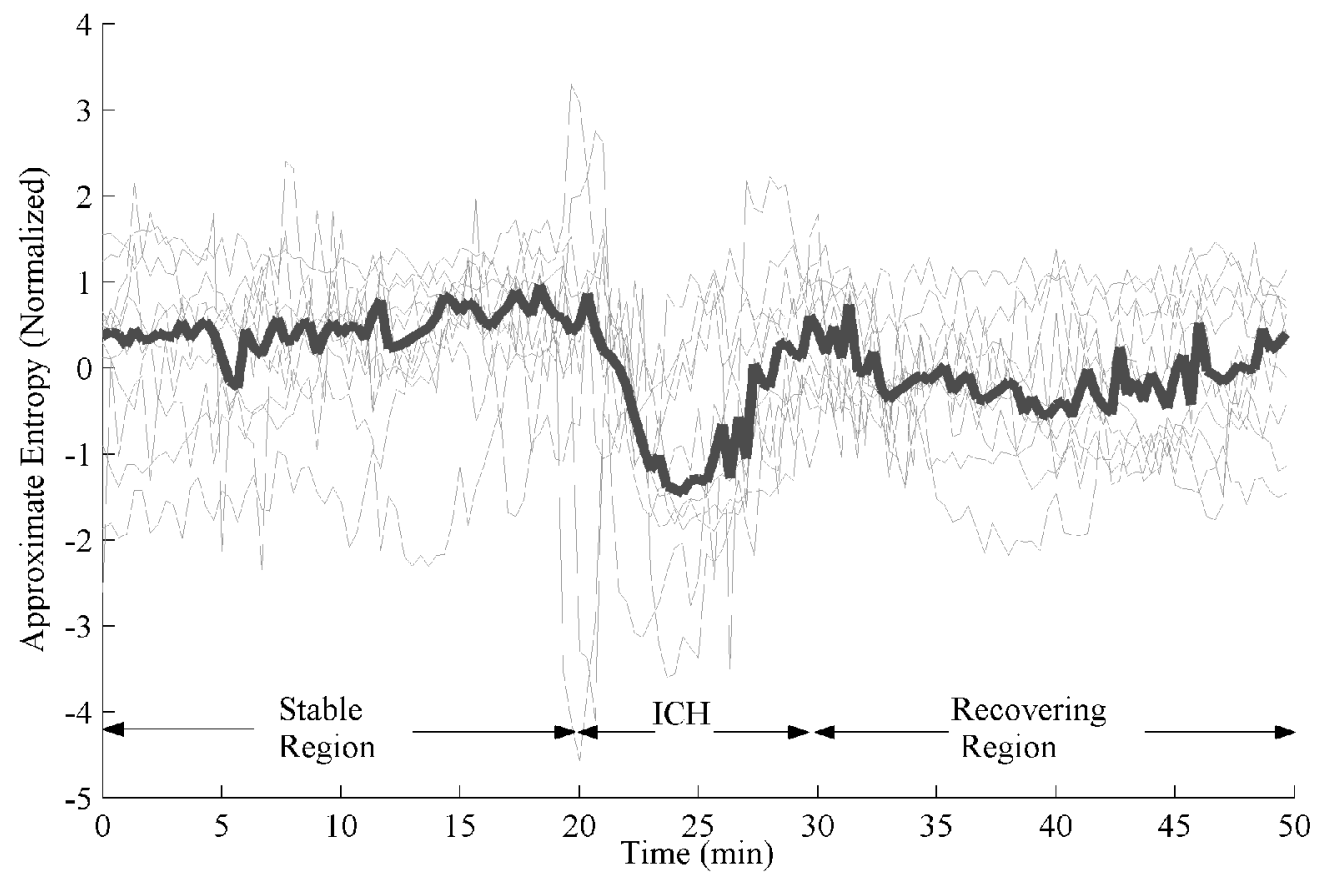

Figure 6.a.

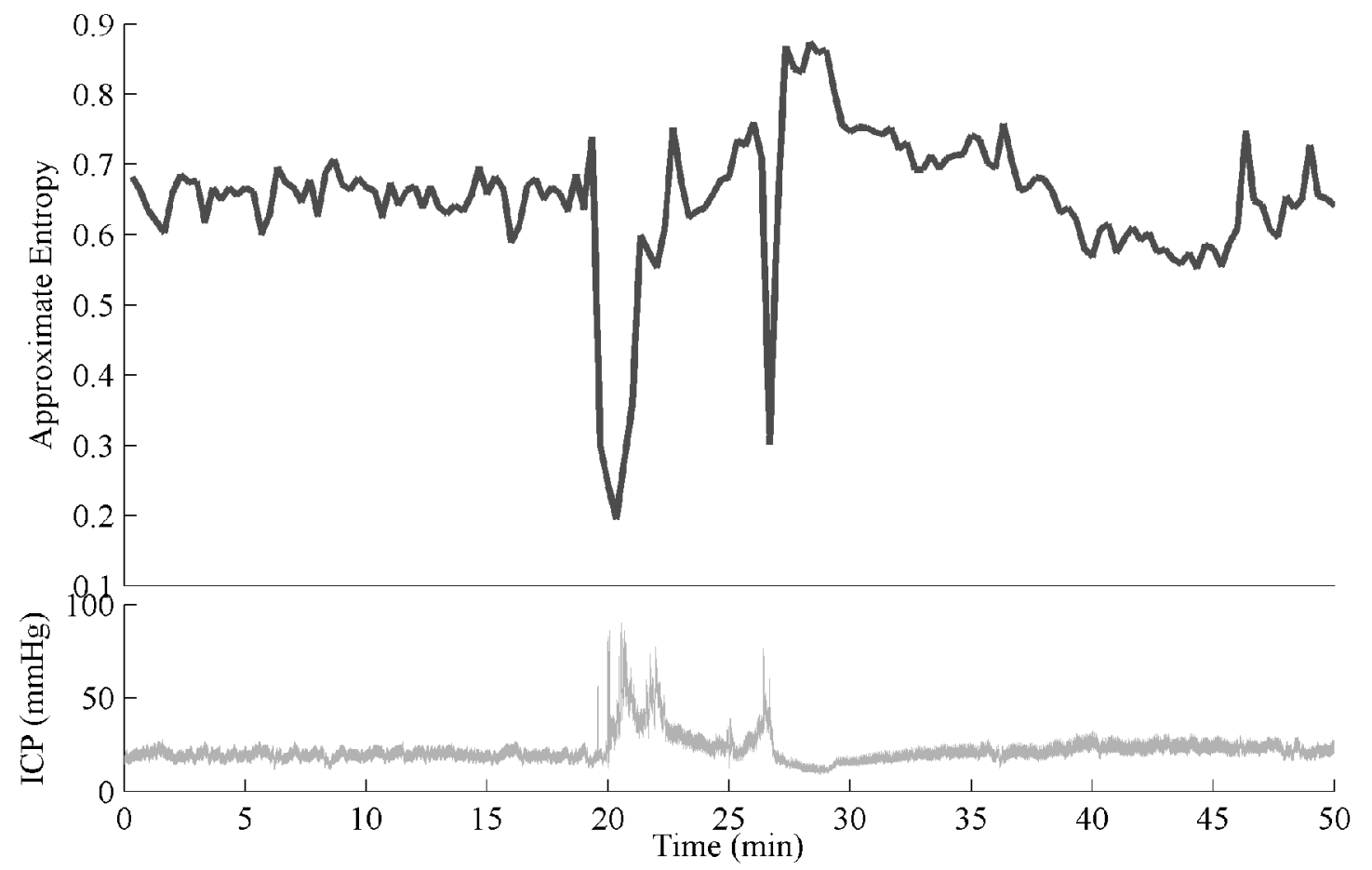


Figure 6.b.

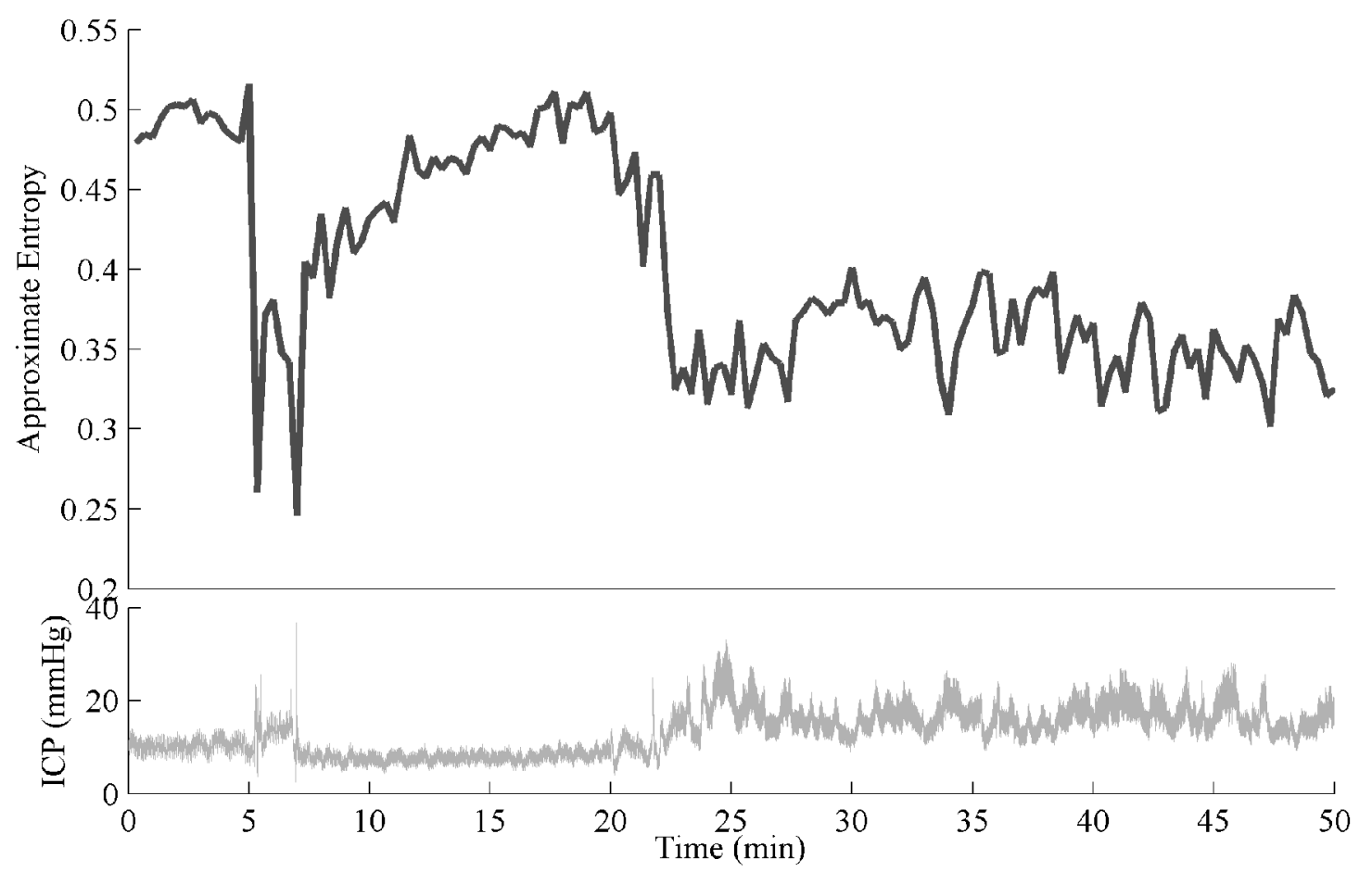

